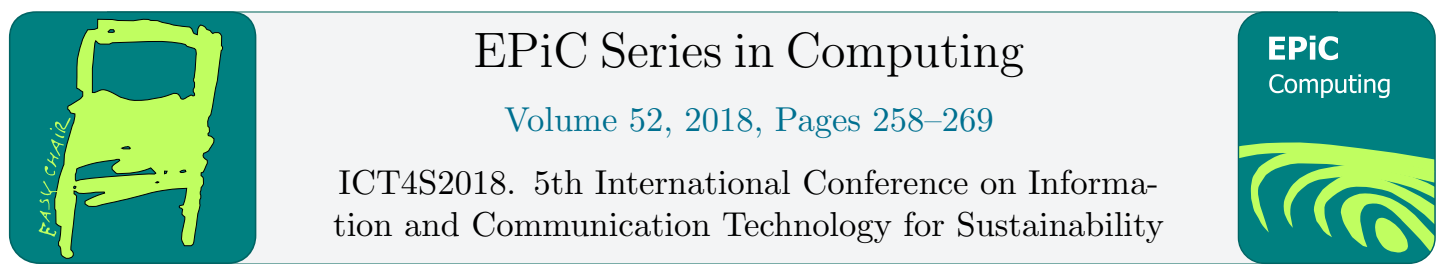

\title{
Shared Autonomous Vehicles: Potentials for a Sustainable Mobility and Risks of Unintended Effects
}

\author{
Christina Pakusch ${ }^{1}$, Gunnar Stevens ${ }^{1,2}$ and Paul Bossauer ${ }^{1}$ \\ ${ }^{1}$ Bonn-Rhein-Sieg University, Sankt Augustin, Germany \\ ${ }^{2}$ University of Siegen, Siegen, Germany \\ \{christina.pakusch; gunnar.stevens; paul.bossauer\}@h-brs.de
}

\begin{abstract}
Automated and connected cars could significantly reduce congestion and emissions through a more efficient flow of traffic and a reduction in the number of vehicles. An increase in demand for driving with autonomous vehicles is also conceivable due to higher comfort and improved quality of time using driverless cars. So far, empirical evidence supporting this hypothesis is missing. To analyze the influence of autonomous driving on mobility behavior and to uncover user preferences, which serve as an indicator for future travel mode choices, we conducted an online survey with a paired comparison of current and future travel modes with 302 German participants. The results do not confirm the hypothesis that ownership will become an outdated model in the future. Instead they suggest that private cars, whether traditional or fully automated, will remain the preferred travel mode. At the same time, carsharing will benefit from full automation more than private cars. However, findings indicate that the growth of carsharing will mainly be at the expense of public transport, showing that more effort should be placed in making public transportation more attractive if sustainable mobility is to be developed.
\end{abstract}

\section{Introduction}

Modern societies are highly mobile with transport being the instrument that enables this user mobility. The increasing need for mobility in the past led to an increase in traffic, especially in urban areas. This development continuously has led to the local and global environmental problems known today: congestion, traffic noise, land sealing, air pollution and $\mathrm{CO} 2$ emissions. Environmental policy therefore calls for the development of sustainable mobility [22]. The sustainable integration of ICT into the value chain is one of the central challenges for the automotive industry to create a future competitive advantage. A radical development from traditional to connected and autonomous driving (AD) can 
already be observed. Various authors assume that the technical innovations in this area will make an important contribution to sustainable mobility: Intelligent traffic management systems and vehicle platooning, so the expectation, will lead to energy savings, congestion can be avoided, traffic flow will become more efficient and driving behavior more ecological. Another expectation is that AD can make the breakthrough for post-ownership mobility [36], with on-demand services such as shared autonomous vehicles (SAV) becoming the dominate travel mode [5,23].

However, innovation history has shown that isolated consideration of the effects of technological efficiency improvements is insufficient. Repeatedly, technical progress has fallen short of expectation as user behavior is not included in relevant research and direct and indirect rebound effects such as consumption shifting towards less emission-friendly travel modes are being neglected. In this regard, we see the danger that the current discourse on $\mathrm{AD}$ primarily underlines the potential benefits of an isolated technical perspective and neglects possible negative effects due to acceptance problems and rebound effects. However, dealing with possible ecological downsides requires more understanding of the effects of new forms of mobility on mobility behavior and of where supplementary measures may be needed to avoid negative effects.

This paper contributes to the current discourse on $\mathrm{AD}$ from a more critical stance taking user behavior as the focus. We conducted an empirical study to include the user into research on travel mode choice that takes future travel modes into consideration, as researchers have requested [26]. Using paired comparison, we analyze user preferences to anticipate changes in mobility behavior, not by using monomodal analysis, as has so far been done [31], but by using multimodal analysis setting different travel modes into relation.

\section{Environmental Impacts of Autonomous Driving}

In the last few years, enormous efforts have been put into enabling AD. SAE International defined six levels of automation for road traffic: no automation (0), driver assistance (1), partial automation (2), conditional automation (3), high automation (4) and the last stage of full automation (5) [34]. In our study we always refer to level 5; full automation. Completely autonomous driving robots that do not have a manual driving mode are expected to be available from 2030 onwards. Optimistic forecasts assume that highly and fully automated vehicles will reach a market share of 11 to 42 percent in 2035 [39].

This rapid progress in the digitalization of the car raises the question of how to assess this development from a sustainability perspective. However, it is important to note that the technology itself is neither eco-friendly nor its opposite. It always depends on its impact on transportation e.g. on traffic system performance or travel behavior. We therefore outline the expected impacts as discussed in the literature.

\subsection{Positive Environmental Effects}

\section{a) Positive Impacts on Traffic System Performance}

$\mathrm{AD}$ would allow vehicle emissions to be reduced by up to 94\% [17]. Connected driving can prevent, reduce or avoid stop-and-go traffic and congestion $[13,36]$. The so-called platooning (travelling close together at high speed) can be used to improve traffic safety, optimize traffic flow, and reduce $\mathrm{CO} 2$ emissions, since vehicles can exchange data with each other and coordinate driving speed, braking characteristics and distances between vehicles [7,40]. Thus, energy consumption could be reduced [7]. As accidents are avoided by using autonomous and connected cars, vehicles require lower safety standards such as airbags and steel constructions than today and would therefore lose weight $[2,4]$. The 
engine performance can then be reduced, which would again lead to lower energy consumption and lower emissions and consequently to a considerable reduction in environmental pollution.

\section{b) Positive Impacts on Travel Mode Choice}

Many researchers expect a greater shift away from private cars towards on-demand mobility services $[14,18]$. Full automation could greatly increase the market share of individual transport such as taxis and carsharing, since the advantages of these business models can be realized to a new extent through autonomous vehicles (AV). Fully autonomous carsharing fleets enable a high degree of spontaneity and flexibility because there are no longer any set arrival and departure times, thus allowing direct competition with private cars [36]. Some studies simulate different scenarios with SAV fleets calculating the number of vehicles needed to ensure efficient mobility with short waiting times and high quality at low costs. They show that SAVs could potentially reduce the number of vehicles by between 31 to 95 percent $[5,8,9,13,14,36]$.

\subsection{Negative Environmental Effects}

While often only ecological, economic and social advantages are discussed, some studies also attempt to assess negative consequences of $\mathrm{AD}$. Researchers are trying to determine how mobility demand will change with the introduction of AVs. Studies such as [5,39] conclude that the widespread use of AVs will increase the number of trips leading to $3 \%$ to $27 \%$ additional journeys [26]. There are various reasons for an increased trip demand [12], some of which are outlined below.

\section{a) Negative Impacts of Empty Runs}

A whole new class of travel will occur with AV maneuvering of unoccupied vehicles [25,39]. AVs can pick up their users, park, and carry out courier journeys autonomously. These additional journeys will occur regardless of whether they are private or shared AVs. With an increase in demand for SAVs, there will be $8 \%$ to $17 \%$ more vehicle-miles traveled for relocation of vehicles or arrival and departure reasons $[5,12,14]$.

\section{b) Negative Impacts of Increased Travel Demand}

As SAV trip costs might be substantially lower than today`s taxi costs [9,14], user demand could rise when trips that, at the moment, have marginal net benefits will become more attractive. In addition, when time during a trip with an AV can be better or more efficiently used, users could consequently be willing to undertake more and longer trips [7]. Such time rebound effects can be caused not only by better use of time but also by the more efficient flow of traffic. Studies have shown that the mobility time budget of users, the amount of time they are prepared to use for travel, is stable over time. So, if an alternative mobility option becomes faster, users will accept longer journeys as the journey time will not place extra demands on their mobility time budget [21].

\section{c) Negative Impacts of Unintended Shifts in Mode Choice}

Most of the simulation models mentioned above are based on the untested assumption that SAVs are especially attractive for car owners, neglecting the fact that they could also unintendedly impact the mobility behavior of other, currently non-car, users. Some studies discuss possible mobility shifts away from eco-friendly mass transportation towards more comfortable SAV offers [39]. Krueger et al. [23], for example, pinpoint that people might prefer inexpensive and convenient mobility on-demand services over previous alternatives such as walking, cycling, or public transport (PT). They fear that PT could be eroded. So far - to our knowledge - these hypotheses have only been argumentatively derived and not empirically tested. We therefore used the travel mode choice theory to systematically check whether this fear is justified and examine these hypotheses empirically. 


\section{Theory of Travel Mode Choice}

Travel mode choice theories aim to predict what transportation mode will be used by understanding why people prefer one mode over another [33]. In general, people have various alternatives for managing mobility and they must choose one of these modes that meets their mobility needs, maximizing personal benefits.

However, users are not able to keep track of all information important for a rational choice [20,35]. The subjective perception of the user therefore does not necessarily have to correspond to objective reality. A mode's benefits only influence mode choice if they are perceived and valued by the users. If, for example, people do not believe that driving by bus is cheaper than by car even if this is the case, they will not choose the bus. As this subjective perception determines users' choice, the bus does not provide a perceived benefit in terms of costs.

In decision making, the absolute level of utility of a good is somehow irrelevant, but differences in utility matter [38]: A travel mode is used not because of its utility in absolute terms, but because its utility is relatively better compared to other alternatives. In the case of adopting new alternative travel modes, these modes must therefore offer a relative advantage over the travel modes previously used.

The random utility theory assumes that the use of alternatives is a latent construct that exists (if at all) in the minds of individuals. According to this theory, people do not compare various goods in general but their attributes that provide (part-worth) utility [24]. In this context, transportation research has identified several travel mode characteristics that affect individual travel mode choice [33]. The following factors are usually mentioned as the most relevant [23,28,37]:

Travel time is the total time required to cover a distance from A to B. Depending on the travel mode, travel time includes not only the actual riding time but also various components for finding a parking space, walking times from an origin to the access point, transfer times, or waiting times at stops.

Travel costs include all costs for the use of a travel mode. For users, it is often only variable costs or perceived costs that are relevant [16].

Comfort includes all the features that make a ride pleasant for the user. Thus, comfort consists of several sub attributes such as the quality of seats, probability of sitting and of transporting goods, the passenger`s privacy, level of crowdedness, high time quality due to good time utilization.

Flexibility refers to the possibility to use the travel mode at your own discretion and adapt it to your own needs.

Availability of the travel modes is given if it is accessible to a user and ready for operation when the user wants or needs it.

Reliability refers to the extent to which a travel mode carries out a trip as intended or scheduled.

Safety describes the condition that gives the passenger the feeling of confidence that a travel mode will take him or her safely to a destination.

In the discourse of $\mathrm{AD}$, the criteria of driving ability, control, and driving fun are repeatedly discussed $[19,29,30]$. AD opens up the possibility of being mobile even when people are temporarily or permanently unable to drive. Many users and researchers see this aspect as an advantage of AD [31]. In contrast, acceptance studies show that users fear loss of control and driving fun when thinking of AD [19].

With a view to encouraging sustainable mobility, studies found environment-related attitudes hardly affect mobility behavior and mode choice in everyday life [15,28].

Taking these factors into account, we evaluate autonomous travel modes and derive first hypotheses on travel mode choice. As direct inclusion of users has been overlooked in research of part-worth utilities of autonomous travel modes, we need to base our estimates of part-worth utilities on general insights from literature. 


\subsection{Changes in Private Car Characteristics}

Some attributes such as travel time, comfort, and time utilization will be improved by automating the car. Travel time is supposed to decrease as connected cars can anticipate and avoid congestion and drive in platoons [26,28]. Driving comfort and convenience are going to increase as travel time can be better used, e.g., for working, relaxing, or socializing and as the car is going to chauffeur its owner and park itself $[9,39,40]$.

Other characteristics such as transportation of goods, personal independence, availability and flexibility will not change significantly [43], as AVs can be used to the same extent to transport goods and as users still have access to their property at all times.

There could also be a deterioration concerning some other characteristics. Users fear that system failures could cause AVs not to run reliably and expect higher acquisition and operating costs from AV technology [19]. In addition, loss of control and driving fun as well as lower safety are negatively linked to AD [19,29-31]. As there seem to be more disadvantages than advantages we conclude that

H1: Users will prefer the traditional car over the fully autonomous car.

\subsection{Changes in Carsharing Characteristics}

Carsharing will benefit from automation in various respects. Travel time will shorten compared to today`s carsharing since the user does not have to walk or use PT to get to the pick-up point. The pure riding time will therefore be similar to that of a (fully automated) private car, apart from the ordering and waiting time. Additionally, automatic relocation of SAVs and automatic pick-up will improve availability and therefore flexibility, too, leveraging personal independence [5,14]. However, perceived availability is still lower than with a privately-owned car, since it cannot be guaranteed that an SAV is always ready to leave [43]. Hauling goods will generally become easier, as passengers will be picked up at their origin and do not need to carry goods to the pick-up point. As in autonomous private cars, time usage, comfort and overall convenience will increase, too. Simulations show that SAV travel costs could be greatly reduced compared to today`s costs of carsharing or taxis [14,18]. However, since most users do not know these simulations and many users are uncertain about the costs of carsharing anyway, this expected cost reduction is probably not relevant for them.

Although there is no knowledge on the users` opinions of safety, reliability, loss of control, and driving pleasure concerning SAVs, we expect that the attitudes that apply to the private AVs also apply to SAVs. However, we think that the loss of control and driving fun is less serious than with a private car as carsharing users might be less emotionally connected to the car. As there seem to be more advantages than disadvantages for carsharing caused by automation, we conclude that

H2: Users will prefer autonomous carsharing (SAV) over traditional carsharing.

Concluding our state of the art, new modes based on AD (such as private AV or SAV) could in the future expand the alternatives in travel modes. The question therefore arises which travel mode users prefer and how this choice will change mobility behavior overall. Although there are many acceptance studies on AD, these studies generally consider the autonomous car in isolation. To our knowledge, travel mode choice analyses have not so far included the new modes, nor have these modes been evaluated in comparison with existing modes of transport.

\section{Methodology}

To investigate the impact of full automation on travel mode choice, an online survey was performed in Germany. We used total paired comparison as a method to uncover user preferences since user preferences are an indicator of future travel mode choice [11,32]. The paired comparison is often used 
when subjective criteria need to be recorded [6]. As already mentioned, this is the case when choosing travel modes, as the actual preference for a travel mode results from the subjectively perceived partworth utilities.

The questionnaire consisted of three parts. First, we asked for general participant demographic information and their current mobility behavior. In the second part, the participants were shown a video to familiarize them with $\mathrm{AD}$ in general and in the form of fully automated carsharing. The last part consisted of the complete paired comparison of the travel modes: private car, automated private car, carsharing, automated carsharing and public transport. In this paired comparison, respondents were confronted with pairs selected from an item set and were asked to select the more preferred item from each pair. With $n=5$ items there were $n=(n *(n-1)) / 2=10$ pairs.

We checked the questionnaire in pretests for comprehensibility and revised it accordingly. Subsequently, the survey was advertised in various social networks and online platforms and was activated from 12/16/2016-01/16/2017 and 06/06/2017-07/06/2017. Two survey periods were chosen to exclude seasonal and weather-dependent causes that influence travel mode choice. In total, the survey was completed by 302 participants. The participants were $49 \%$ male and $51 \%$ female. The average age of the respondents was 36.1 years, $57.9 \%$ of them lived in the city and $42.1 \%$ in rural areas. Of the 302 respondents, $97 \%$ held a driving license, $80.1 \%$ owned a car, and $34.4 \%$ held a long-term ticket for PT.

\section{Results}

\subsection{Preferences of the Various Travel Modes}

To provide an aggregate choice matrix for our sample, the individual response matrices are totalized. Table 1 shows the frequency matrix of paired comparison representing the judgements of all participants. The values specify the frequency with which each item was chosen across all paired comparisons. For example, the value 180 or $59.6 \%$ in cell 1,2 means that the traditional private car was preferred over the automated private car by 180 respondents, $59.6 \%$ of the sample, respectively (cell 2,1 shows the equivalent of 122 and 40.4\%). The values are printed in bold if the user preference is greater than 50\%. An asterisk indicates values that deviate significantly from 50\%; a value is significant ( $\mathrm{p}$-value $<0.05$ ) according to a binomial proportion test [27]. To determine the rank, the sum of the columns was formed [6].

\begin{tabular}{|c|c|c|c|c|c|}
\hline & Car & $\begin{array}{c}\text { Automated } \\
\text { Car }\end{array}$ & Carsharing & $\begin{array}{l}\text { Automated } \\
\text { Carsharing }\end{array}$ & $\begin{array}{c}\text { Public } \\
\text { Transport }\end{array}$ \\
\hline & $\operatorname{Num}(\%)$ & $\operatorname{Num}(\%)$ & $\operatorname{Num}(\%)$ & $\operatorname{Num}(\%)$ & $\operatorname{Num}(\%)$ \\
\hline Car & & $122(40.4)^{*}$ & $53(17.5)^{*}$ & $65(21.5)^{*}$ & $79(26.2)^{*}$ \\
\hline Automated Car & $180(59.6)^{*}$ & & $103(34.1)^{*}$ & $78(25.8) *$ & $109(36.1)^{*}$ \\
\hline Carsharing & $249(82.5)^{*}$ & $199(65.9)^{*}$ & & $178(58.9) *$ & $180(59.6)^{*}$ \\
\hline Automated Carsharing & $237(78.5)^{*}$ & $224(74.2)^{*}$ & $124(41.1)^{*}$ & & $142(47.0)$ \\
\hline Public Transport & $223(73.8)^{*}$ & $193(63.9)^{*}$ & $122(40.4)^{*}$ & $160(53.0)$ & \\
\hline Sum & 889 & 738 & 402 & 481 & 510 \\
\hline Utility Scale Value ( \pm CI) & $1.00( \pm 0.08)$ & $0.63( \pm 0.06)$ & $0.24( \pm 0.03)$ & $0.30( \pm 0.03)$ & $0.33( \pm 0.04)$ \\
\hline Ranking & 1 & 2 & 5 & 4 & 3 \\
\hline
\end{tabular}

$*: \mathrm{p} \leq 0.05$

Table 1: Paired Comparison Matrix 


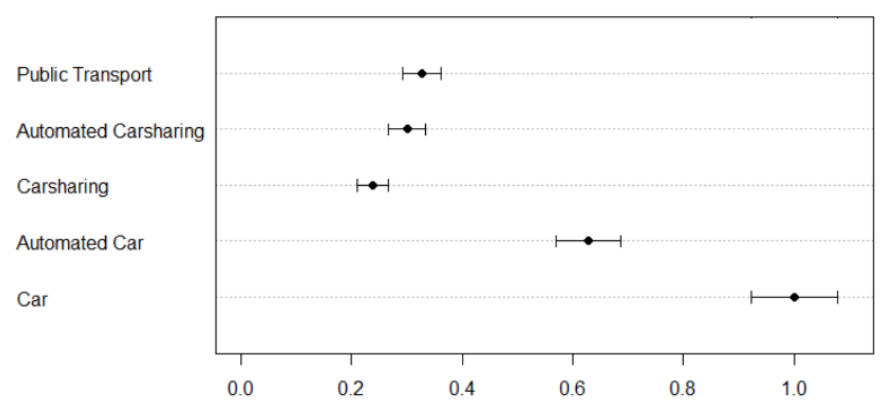

Figure 1: Utility Scale Values (BTL Model)

Participants' appraisals are largely consistent. A result is consistent if a person's ranking is free of contradictions [6]. The mean consistency coefficient of the paired comparative judgements is 0.95 ; most respondents therefore have a conscious or unconscious individual preference order of the presented travel modes.

Overall, results show that the private car has the highest preference in the sample, the fully automated version ranks second, followed by PT while carsharing-based modes finish last with autonomous carsharing still ranking higher than traditional carsharing.

We additionally scaled sum values by applying the Bradley-Terry-Luce (BTL) model as one of the most simple and commonly applied models in the analysis of paired comparison data [10,41]. The resulting utility scale values (USV) provide a ranking of the different modes that reveals relative distances between the individual ranks [1]. Table 1 and Figure 1 show USV and corresponding 95\% confidence intervals for each travel mode. The USV shows that the perceived utility of both the car and the automated car are dominant. It also shows that the perceived utilities of the public and shared modes are close together. They form a group whose value is below that of the private modes. Still, the scale values must be considered with caution. The low goodness of fit $\left(\chi^{2}=19.47\right)$ indicates the BTL model is a simplification concerning the utility structure of the average user, one that abstracts from the difference between preferences of individual users and user segments.

In addition to the one-dimensional utility scale, interesting results can also be seen in the comparison of individual transport modes. For private cars, the direct comparison of fully automated vs. traditional variant shows a significant preference for the traditional car $(59.6 \% ; \mathrm{p}<0.05)$. Thus, there is evidence, that Hypothesis 1 is true. For carsharing, it is exactly the opposite. Participants prefer fully automated carsharing to traditional carsharing $(64.6 \%$; $p<0.05)$ supporting Hypothesis 2.

To see how the offer of autonomous modes affects eco-friendly means of transport such as PT, we look at changes in the preferences of PT compared first with traditional modes and second with respect to autonomous modes. The paired comparison of PT and private cars shows that the preference for PT is $26.2 \%$ compared to the preference for the traditional car and $36.1 \%$ compared to that of the autonomous car. Thus, automating the car does not lead to an increase in preference for the private car, which might have been expected due to advantages that come with automation.

For carsharing it is again the other way around. While $59.6 \%$ of users prefer PT to traditional carsharing, only $47 \%$ prefer PT to autonomous carsharing. The preference for PT decreases when carsharing is automated. In contrast to private cars, carsharing seems to benefit from full automation.

\subsection{Changes in the First-Choice Preference}

With the first-choice rule, the user always chooses the product with the highest overall utility among the alternatives offered [42]. This rule means that the alternative with the highest utility receives a choice probability of 1 , and all other alternatives receive the probability of 0 . In the context of travel 


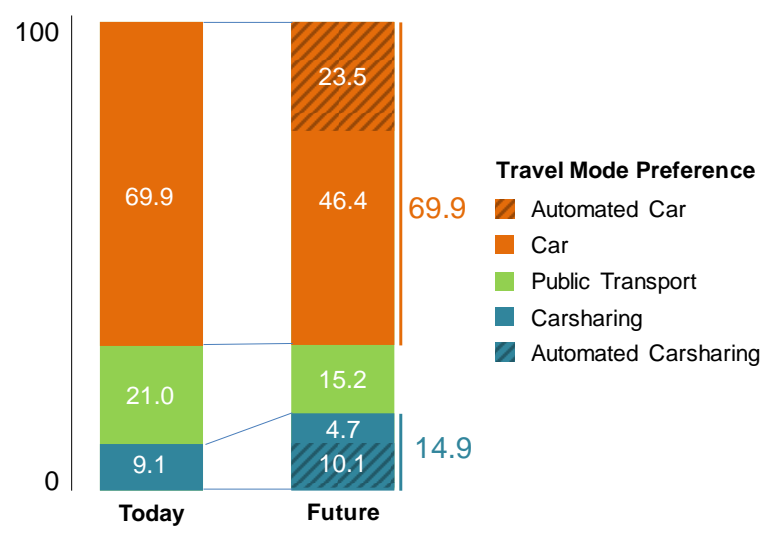

Figure 2: Shift in Mobility Demand

mode choice, first choice analysis is insofar useful as users usually prefer one travel mode that is used regularly as long as circumstances remain relatively stable [3].

To analyze the overall effect of the autonomous modes of transport on the travel mode choice in more detail, we compared the results of the paired comparisons without new modes of transport (private car, carsharing, and PT) with the results of the paired comparisons with new modes of transport (private car, automated private car, carsharing, automated carsharing and PT, Figure 2). When only taking currently available means of transport into account, the participants prefer cars $(69.9 \%)$ over PT $(21.0 \%)$ and carsharing $(9.1 \%)$. If the paired comparisons are expanded by autonomous cars and autonomous carsharing, the respondents' preference structure changes. The proportion of decisions in favor of privately owned cars (traditionally and autonomously) remains respectively stable at $69.9 \%$. There are greater differences in the shares for PT and carsharing. While the share of carsharing (traditionally and autonomously) increases from $9.1 \%$ to $14.9 \%$, the share of PT decreases from $21.0 \%$ to $15.2 \%$.

The figures indeed suggest that carsharing can be strengthened by fully automated systems and that market shares can be gained. According to our participants' choices, however, the desired effect of reducing car ownership in favor of sharing offers is not achieved. Instead, the more intensive use of carsharing is to the detriment of PT.

\section{Discussion}

The (fully automated) private car seems to be of great importance for the respondents also in the future. However, the preference for carsharing-based services and PT could change significantly in the future. To investigate changes in the preference structure, the results of the paired comparisons will be discussed in more detail, considering possible changes in part-worth utilities.

\subsection{The Private Car Remains Most Preferred Mode}

The participants confirm that the traditional private car has a slightly higher total utility than the autonomous private car, so that $59.6 \%$ of the participants still prefer the traditional version. For sure the privately-owned car being the most popular travel mode today affects the preferences for future travel mode choices. As travel mode choice is strongly influenced by socialization and habits, users familiar with using a private car considerably more often choose the private car to be the most preferred travel 
mode in the future, too [3]. From today's view point, the subjectively perceived advantages of the classic car over the autonomous car are predominant. While characteristics such as availability, driving time, flexibility or transportation of objects are not expected to change substantially, other characteristics might be perceived as very different in traditional and autonomous cars. According to some studies, users could see the autonomous car as being more comfortable, better in terms of driving comfort, improved time use and less restrictive (e.g., after drinking alcohol) [31]. In terms of driving enjoyment and control of the vehicle, however, the traditional model that needs to be actively controlled can score better. Studies such as [31] have shown that some respondents fear that automating the car will reduce driving fun. There are also still great reservations about the safety of the still unknown AV [19], so that the partial benefit of safety in traditional passenger cars may be valued higher.

\subsection{Carsharing Strongly Benefits from Automation}

The high value of $64.6 \%$ indicates that the automation of carsharing is widely accepted, because existing disadvantages such as lack of availability, flexibility, having to use more than one mode of transport, and high costs are completely or partially eliminated $[23,39,40]$. Participants seem to be anticipating the benefits of fully automated carsharing. Just as with a private car, comfort and the use of time will increase as the driving task is no longer necessary. In addition, the planning and coordination effort is reduced because the user does not have to search for and locate a suitable vehicle and a station; the vehicle comes to the user on request [43].

When directly comparing the preferences for fully autonomous modes for either the private car or carsharing, it is also noticeable that $58.9 \%$ of the respondents prefer the fully automated version for carsharing, compared to only $40.4 \%$ for private cars. This significant difference in user preferences (binomial proportion test; $\mathrm{p}<0.05$ ) indicates that the acceptance of full automation depends on the increase in total utility of the respective traffic mode. From the user's point of view, full automation in the private car segment therefore brings comparatively minor improvements, while it greatly increases the benefits and thus the attractiveness of carsharing. It is therefore interesting to see how the preferences for full automation shifts between private cars and carsharing. In the traditional scenario there is a significant preference of $82.5 \%$ for private cars. In the fully automated scenario, there is also a significant preference for the private car, but it decreases significantly to $74.2 \%(\mathrm{p}<0.05)$. In other words, the relative total utilities of private cars and carsharing will converge to some extent in the future. It can therefore be assumed that the full automation of carsharing can help to increase market potential and expand its share in the modal split.

\subsection{Public Transport as Loser of Vehicle Automation}

The indirect effects of full automation on PT must also be considered. The paired comparisons of PT with both carsharing variants show that the preference for PT decreases significantly from $59.6 \%$ to $47.0 \%(\mathrm{p}<0.05)$. This decrease can be attributed to a relative improvement in carsharing compared to PT, caused by the automation of carsharing. In this way, the availability and ease of use of carsharing is improved. In addition, driving skills no longer play a role in the use of autonomous carsharing. Using a travel mode without having to be fit for driving is so far one of the unique selling points of PT.

Generally, this improvement also applies to private cars. Surprisingly, the responses related to the fully automated private car show an increase in preference for PT from $26.2 \%$ to $36.1 \%$. Due to the fully automated system, the private car loses attractiveness compared to PT. One explanation for this could be that, due to a lack of confidence in technology, today's drivers would prefer to use PT instead of a fully automated car. 


\section{Limitations and Implications for Future Research}

When considering all results, it is important to note that the study is not representative and that our results can therefore not be directly transferred to the whole population. In addition, it should be noted that the survey was carried out in Germany and that the German transport system and consumer habits certainly differ from those in other countries in various aspects. Such differences may also affect the participants`assessment of future mobility concepts.

The figures on user preferences must be viewed in light of user preferences not reflecting the current modal split, as in particular carsharing has a much smaller market share. However, differences in the theoretical and the actual travel mode choice can be observed repeatedly and still the ranking of market shares is correct. Routines and habits also prevent users from choosing an alternative means of transport [3]. Furthermore, we did not consider automated forms of PT in our paired comparison since a recent study showed that, because there is generally no interaction between driver and passenger in PT, user do not perceive a significant difference between automated and non-automated PT [29].

As mentioned before, the BTL model has a low goodness of fit. Therefore, the resulting utility scale does not hold for the individual user; it only holds for the aggregated average user. In future, user segments that have a more homogeneous preference structure should be studied in more detail. Such segmentation will provide a more differentiated picture. In addition, we only indirectly examined the perceived part-worth utilities by analyzing the travel mode choice and drawing conclusions on the individual part-worth utility. In further research, these part-worth utilities should be investigated empirically.

\section{Conclusion}

Literature often assumes that autonomous driving will have a positive effect on the environmental balance since autonomous vehicles implement fuel-efficient driving strategies such as anticipatory driving and reduce congestion density, congestion frequency, and number of vehicles [14,17]. Unintended rebound effects such as consumption shifting have largely been overlooked in this discourse. In Studies that analyze the downsides of autonomous driving, impacts are assessed from a logical perspective; empirical evidence is still missing. Studies do not take the actual user preference into account by involving the users themselves. However, it is the user who will decide for or against the use of self-driving vehicles in the future and who will adapt his or her mobility behavior to the new offers such as automated carsharing or shared autonomous vehicles.

Our empirical analysis supports the hypotheses that users will prefer first, traditional cars over fully autonomous ones and second, autonomous carsharing over traditional carsharing. Carsharing strongly benefits from automation as it can increase its utility in various ways. However, carsharing is not being strengthened at the expense of private cars as hoped for, but at the expense of public transport.

Since there is evidence that not-environmental-related criteria particularly such as comfort and reliability are decisive for travel mode choice, it is important to strengthen sustainable mobility, which cannot only be done by the users. The government must make a greater effort in transportation policy by making sustainable public transport more attractive for people and providing incentives for private companies to offer sustainable mobility.

\section{References}

[1] Agresti, A., An introduction to categorical data analysis, (Wiley New York, 1996). 
[2] Anderson, J. M., Nidhi, K., Stanley, K. D., Sorensen, P., Samaras, C., and Oluwatola, O. A., Autonomous vehicle technology: A guide for policymakers, (Rand Corporation, 2014).

[3] Bamberg, S., Ajzen, I., and Schmidt, P., Choice of travel mode in the theory of planned behavior: The roles of past behavior, habit, and reasoned action, Basic and Applied Social Psychology 25 (2003) 175-187.

[4] Begg, D., A 2050 vision for London: what are the implications of driverless transport?, (2014).

[5] Bischoff, J., and Maciejewski, M., Simulation of city-wide replacement of private cars with autonomous taxis in Berlin, Procedia Computer Science 83 (2016) 237-244.

[6] Bortz, J., and Döring, N., Forschungsmethoden und evaluation, (Springer-Verlag, 2013).

[7] Brown, A., Gonder, J., and Repac, B., An analysis of possible energy impacts of automated vehicle, in Road Vehicle Automation, (Springer, 2014), pp. 137-153.

[8] Burghout, W., Rigole, P. J., and Andreasson, I., Impacts of shared autonomous taxis in a metropolitan area, in Proceedings of the 94th Annual Meeting of the Transportation Research Board, Washington DC, 2015, 2015).

[9] Burns, L. D., Sustainable mobility: a vision of our transport future, Nature 497 (2013) 181-182.

[10] Cattelan, M., Models for paired comparison data: A review with emphasis on dependent data, Statistical Science (2012) 412-433.

[11] David, H. A., The method of paired comparisons, (London, 1963).

[12] Davidson, P., and Spinoulas, A., Driving alone versus riding together-How shared autonomous vehicles can change the way we drive, Road \& Transport Research: A Journal of Australian and New Zealand Research and Practice 25 (2016) 51.

[13] Fagnant, D. J., and Kockelman, K., Preparing a nation for autonomous vehicles: opportunities, barriers and policy recommendations, Transportation Research Part A: Policy and Practice 77 (2015) 167-181.

[14] Fagnant, D. J., Kockelman, K. M., and Bansal, P., Operations of Shared Autonomous Vehicle Fleet for the Austin, Texas Market, Transportation Research Record: Journal of the Transportation Research Board (2015) 98-106.

[15] Franzen, A., Umweltbewusstsein und Verkehrsverhalten-Empirische Analysen zur Verkehrsmittelwahl und der Akzeptanz umweltpolitischer Massnahmen, (1997).

[16] Gardner, B., and Abraham, C., What drives car use? A grounded theory analysis of commuters' reasons for driving, Transportation Research Part F: Traffic Psychology and Behaviour 10 (2007) 187-200.

[17] Greenblatt, J. B., and Saxena, S., Autonomous taxis could greatly reduce greenhouse-gas emissions of US light-duty vehicles, Nature Climate Change 5 (2015) 860-863.

[18] Greenblatt, J. B., and Shaheen, S., Automated vehicles, on-demand mobility, and environmental impacts, Current Sustainable/renewable Energy Reports 2 (2015) 74-81.

[19] Howard, D., and Dai, D., Public perceptions of self-driving cars: The case of Berkeley, California, in Transportation Research Board 93rd Annual Meeting, 2014).

[20] Jou, R.-C., Hensher, D. A., Liu, Y.-H., and Chiu, C.-S., Urban commuters' mode-switching behaviour in Taipai, with an application of the bounded rationality principle, Urban Studies 47 (2010) 650-665.

[21] KIT (Institut für Verkehrswesen, Karlsruher Institut für Technologie), German Mobility Panel, 2017).

[22] Kramers, A., Höjer, M., Lövehagen, N., and Wangel, J., ICT for Sustainable Cities: How ICT can support an... - Google Scholar, in Proceedings of the First International Conference on Information and Communication Technologies for Sustainability, ETH Zürich, 2013), pp. 183188.

[23] Krueger, R., Rashidi, T. H., and Rose, J. M., Preferences for shared autonomous vehicles, Transportation Research Part C: Emerging Technologies 69 (2016) 343-355. 
[24] Lancaster, K. J., A new approach to consumer theory, Journal of Political Economy 74 (1966) 132-157.

[25] Litman, T., Autonomous vehicle implementation predictions, Victoria Transport Policy Institute 28 (2017).

[26] Milakis, D., Van Arem, B., and Van Wee, B., Policy and society related implications of automated driving: A review of literature and directions for future research, Journal of Intelligent Transportation Systems (2017) 1-25.

[27] NIST (Ed.), Binomial Proportion Test, (2015).

[28] Owczarzak, ILukasz, and Żak, J., Design of passenger public transportation solutions based on autonomous vehicles and their multiple criteria comparison with traditional forms of passenger transportation, Transportation Research Procedia 10 (2015) 472-482.

[29] Pakusch, C., and Bossauer, P., User Acceptance of Fully Autonomous Public Transport, in Proceedings of the 14th International Joint Conference on E-Business and Telecommunications (ICETE 2017), 2017), pp. 52-60.

[30] Pakusch, C., Bossauer, P., Shakoor, M., and Stevens, G., Using, Sharing, and Owning Smart Cars, in Proceedings of the 13th International Joint Conference on E-Business and Telecommunications (ICETE 2016), 2016), pp. 19-30.

[31] Payre, W., Cestac, J., and Delhomme, P., Intention to use a fully automated car: Attitudes and a priori acceptability, Transportation Research Part F: Traffic Psychology and Behaviour 27 (2014) 252-263.

[32] Rahman, F., Yoshida, S., Kojima, A., and Kubota, H., Paired Comparison Method to Prioritize Traffic Calming Projects, Journal of the Eastern Asia Society for Transportation Studies 11 (2015) 2472-2487.

[33] Ravi Sekhar, C., Mode Choice Analysis: The Data, the Models and Future Ahead, International Journal for Traffic and Transport Engineering 4 (2014).

[34] SAE International (Ed.), Automated Driving - Levels of Driving Automation are Defined in NEW SAE International Standard J3016, (2016).

[35] Simon, H. A., A behavioral model of rational choice, The Quarterly Journal of Economics 69 (1955) 99-118.

[36] Spieser, K., Treleaven, K., Zhang, R., Frazzoli, E., Morton, D., and Pavone, M., Toward a systematic approach to the design and evaluation of automated mobility-on-demand systems: A case study in Singapore, in Road Vehicle Automation, (Springer, 2014), pp. 229-245.

[37] Steg, L., Can public transport compete with the private car?, IATSS Research 27 (2003) 27-35.

[38] Train, K. E., Discrete choice methods with simulation, (Cambridge university press, 2009).

[39] Trommer, S., Kolarova, V., Fraedrich, E., Kröger, L., Kickhöfer, B., Kuhnimhof, T., et al., Autonomous Driving-The Impact of Vehicle Automation on Mobility Behaviour, (2016).

[40] Walker, W. E., and Marchau, V. A., Dynamic adaptive policymaking for the sustainable city: The case of automated taxis, International Journal of Transportation Science and Technology 6 (2017) $1-12$.

[41] Wickelmaier, F., and Schmid, C., A Matlab function to estimate choice model parameters from paired-comparison data, Behavior Research Methods, Instruments, \& Computers 36 (2004) 2940.

[42] Wittink, D. R., and Cattin, P., Commercial use of conjoint analysis: An update, The Journal of Marketing (1989) 91-96.

[43] Wödl, S., Pakusch, C., Bossauer, P., and Stevens, G., Auswirkungen vollautomatisierter PKWs auf die Verkehrsmittelwahl, Internationales Verkehrswesen 69 (2017) 68-72. 